\title{
Review of Current Practices of Peripheral Nerve Blocks for Hip Fracture and Surgery
}

\author{
Jyoti Dangle $^{1} \cdot$ Promil Kukreja $^{1} \cdot$ Hari Kalagara ${ }^{1}$ \\ Published online: 26 May 2020 \\ (C) The Author(s) 2020
}

\begin{abstract}
Purpose of Review This article aims to describe the anatomical and technical aspects of various regional techniques used for fracture hip and hip surgery. We reviewed the commonly used nerve blocks, interfascial plane blocks and current evidence of their utility in hip fracture patients.

Recent Findings Fascia iliaca compartment block (FICB) and femoral nerve block (FNB) are the most commonly used nerve blocks for providing pain relief for hip fracture patients. Supra-inguinal FICB has more consistent spread to all nerves and can enable better pain control. Both the FICB and FNB have shown analgesic efficacy with reduced pain scores, opioid sparing effect, and they enable better patient positioning for spinal in the operating room. These nerve blocks in the elderly patients can also have beneficial effects on delirium, reduced hospital length of stay, and decreased incidence of pneumonia. Some of the novel interfascial plane blocks like PEricapsular Nerve Group (PENG) blocks are now being explored to provide pain relief for fracture hip.

Summary Hip fracture in the elderly has associated morbidity and mortality. Early surgical intervention has shown to reduce morbidity and mortality. Pain management in this elderly population poses a unique challenge and complementing with regional anesthesia for analgesia has shown numerous benefits.
\end{abstract}

Keywords Hip fracture $\cdot$ Peripheral nerve blocks $\cdot$ Regional anesthesia $\cdot$ Fascia iliaca block $\cdot$ Femoral nerve block $\cdot$ PENG block

\section{Introduction}

The current shift toward longevity brings with it a multitude of medical issues. One such is the incidence of osteoporotic fractures, namely, hip fracture. The annual incidence of hip fractures in the USA is 280,000 with women being twice as prone as men especially in the elderly population [1]. A comparison study in postmenopausal women showed that the burden of hospital cost was the highest for hospitalizations due to osteoporotic fractures with myocardial infarction ranking in second followed by stroke and then breast cancer [2]. Studies have shown that hip fractures in elderly are associated with significant morbidity, especially the need of long-term care

This article is part of the Topical Collection on Regional Anesthesia

Jyoti Dangle

jdangle@uabmc.edu

1 Department of Anesthesiology and Perioperative Medicine, University of Alabama at Birmingham (UAB), Birmingham, AL, USA and 1-year mortality of $30 \%$. Opioids are commonly used along with other multimodal techniques to address pain. The elderly due to altered pharmacodynamics and coexisting medical conditions are vulnerable to the side effects of opioids especially opioid-related respiratory depression. Early surgery within $48 \mathrm{~h}$ of fracture has shown to decrease complication and mortality rates [3]. This gives an opportunity for anesthesiologists to participate in patient's pain control early on especially in terms of offering regional analgesia for effective pain control. The nerve blocks provide superior analgesia with decreased use of opioids and various other benefits. In this current review, we have described the commonly used nerve blocks like fascia iliaca, femoral, and some novel interfascial plane blocks for hip fracture analgesia.

\section{Anatomical Background}

The hip joint is a classic ball and socket joint formed by the acetabulum and the head of the femur. The hip joint is innervated by both lumbar (L1-L4) and sacral (L4-S4) plexuses, 
and receives its sensory innervation from the femoral, obturator, and sciatic nerves with contribution from nerve to quadratus femoris and superior gluteal nerve [4]. Cutaneous innervation is by lateral femoral cutaneous, genitofemoral, and obturator nerves. A variety of nerve blocks have been utilized for hip fracture, namely, lumbar plexus block, fascia iliaca block, femoral block, sciatic block, and 3 in 1 block. In recent times, novel interfascial plane blocks like Quadratus Lumborum Block (QLB) and PEricapsular Nerve Group Block (PENG) have been used for analgesia. In this review, we have described the anatomical basis, technical aspects, and evidence of various nerve blocks used for hip fracture and hip surgery analgesia.

\section{Fascia Iliaca Block: (FIB/FICB)}

The fascia iliaca compartment block (FICB) is a block where large volume of dilute local anesthetic medication is deposited deep to the fascia iliaca. This fascia covers the anterior surface of iliacus and psoas muscles and creates a compartment between its attachments to the iliac crest laterally and psoas fascia medially and the muscles it covers. Local anesthetic (LA) deposited in this compartment is assumed to spread to the femoral, obturator and lateral femoral cutaneous (LFCN) nerves.

\section{Loss of Resistance Technique}

Dalens et al. described the loss of resistance technique. In this technique, a line is drawn from the anterior superior iliac spine to pubic tubercle. This line is divided into three parts, and the needle is inserted at the junction of middle and lateral thirds. The needle is inserted perpendicular to the skin till a double popping sensation is felt which correlates to the needle penetrating the fascia lata and then fascia iliaca. LA is deposited in the tissue plane after the second pop is felt. This technique can have high failure rates due to subjective feeling of the fascia with pops and higher complication rates compared with the ultrasound guided technique.

\section{Ultrasound Guided Technique}

The ultrasound technique is now widely used and ensures deposition of LA in the correct fascial plane. In this technique, a high frequency linear probe is placed transversely at the level of the inguinal crease (Fig. 1). After the femoral artery is identified, the probe is moved laterally, and the hypoechoic iliopsoas muscle is identified which is covered by the hyperechoic fascia iliaca (Fig. 1b). This fascia invests the femoral nerve medially and laterally covers the sartorius muscle. A needle is inserted in-plane from lateral to medial and pierces the fascia iliaca at the junction of medial border of sartorius and iliopsoas muscle. The LA is deposited deep to

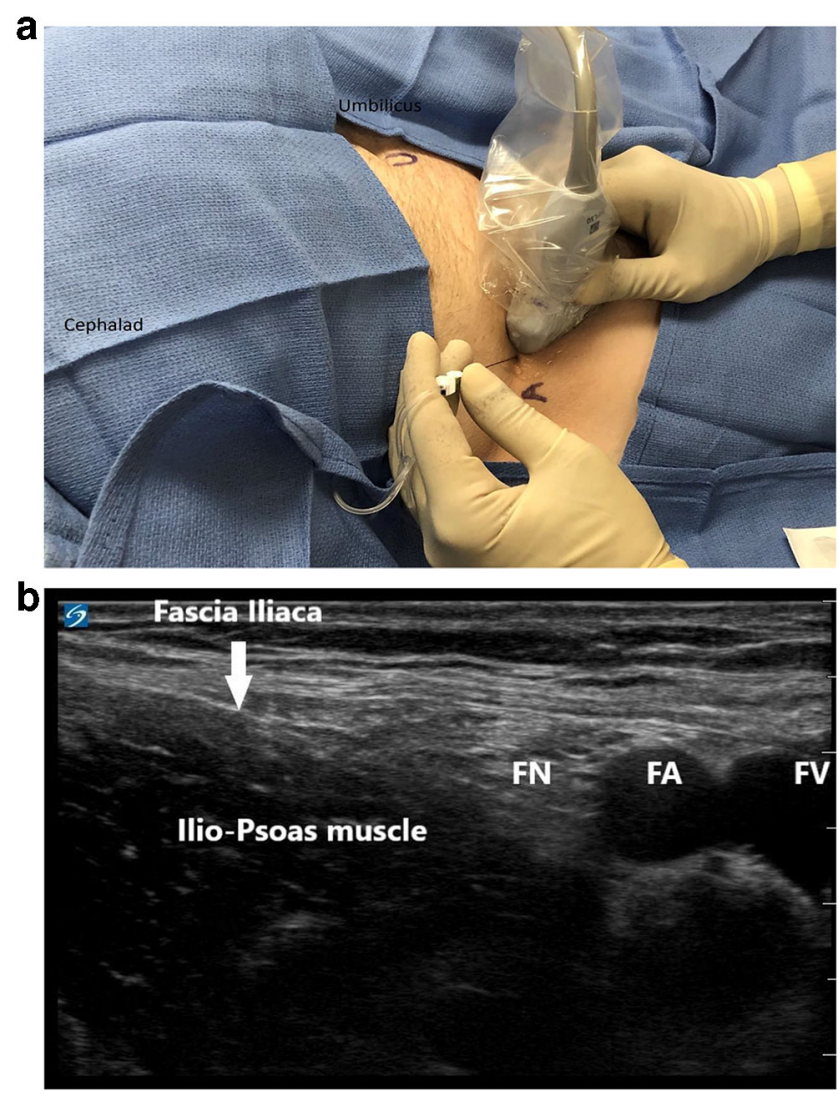

Fig. 1 a Infra-inguinal fascia iliaca block and femoral nerve block. The image shows the probe position at the inguinal crease and in-plane approach of the needle from lateral to medial. b Sono-anatomy at the inguinal crease showing the fascia iliaca, femoral nerve (FN), femoral artery (FA), femoral vein (FV), and iliopsoas muscle

fascia iliaca and should be seen spreading toward the femoral nerve medially. This block can be performed as a single shot or as a continuous catheter technique for prolonged analgesia. This is described as the infra-inguinal FIB technique (IIFIB).

Hebbard et al. described a new ultrasound guided suprainguinal approach (SIFIB) [5]. A high frequency linear probe is placed over the inguinal ligament in the parasagittal plane close to the anterior superior iliac spine. Here the hyperechoic line of ilium is identified covered by the echolucent iliacus muscle with the fascia iliaca covering the muscle (Fig. 2). The probe is moved supero-laterally to identify the anterior superior iliac spine. The deep circumflex iliac artery should be identified superficial to the fascia iliaca. The needle is introduced with in-plane approach till it penetrates through the fascia iliaca, and LA is seen spreading over the iliacus muscle into iliac fossa. A modification of this approach has been described by Desmet et al., where the probe is tilted with cranial end toward umbilicus and caudal end toward anterior superior iliac spine (Fig. 3) [6]. Here the bow tie formed by muscle fasciae of sartorius, iliopsoas, and intra-abdominal oblique muscle is identified (Fig. 3b). The needle is inserted just above the inguinal crease, in an in-plane approach and 
Fig. 2 Sono-anatomy of the supra-inguinal approach to fascia iliaca block showing the fascia iliaca (noted by white arrows) covering the iliacus muscle at level of anterior superior iliac spine (ASIS)

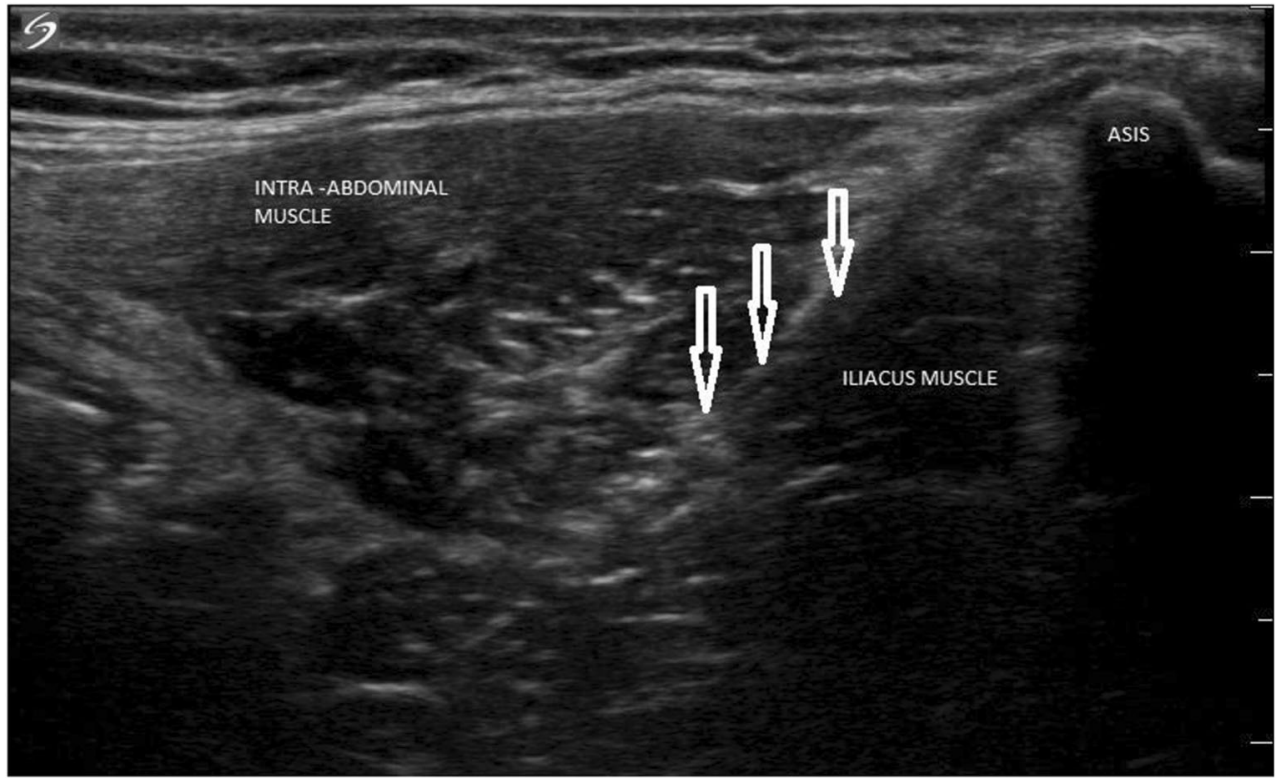

a
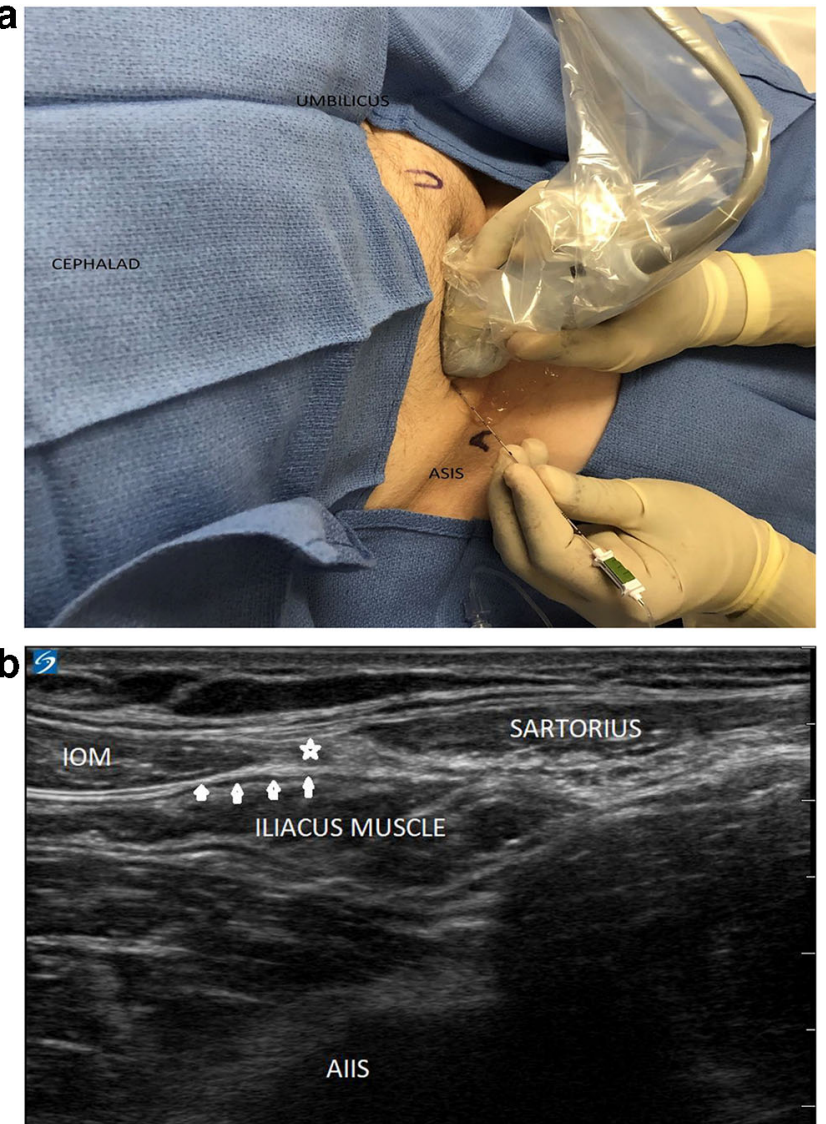

Fig. 3 a Supra-inguinal fascia iliaca block. The image shows the probe position above the inguinal crease, with cranial end of probe towards umbilicus and caudal end toward anterior superior iliac spine (ASIS) showing the bowtie as seen in Fig. 3b. The needle is inserted in-plane from lateral to medial. b Sono-anatomy of supra-inguinal approach to fascia iliaca block (denoted by arrows) showing the bow tie $(\star)$ formed by muscle fasciae of sartorius and internal oblique muscle (IOM). AIIS is anterior inferior iliac spine hydro-dissection performed at the junction of bow tie to see the spread of LA in the fascial plane above the iliacus muscle.

The SIFIB approach has shown consistent spread to the three nerves of the lumbar plexus, which contribute to the sensory supply of hip joint [7•]. Both infra and suprainguinal approaches of FICB with dilute LA have shown to preserve motor strength of the lower extremity [7•].

\section{Analgesic Efficacy}

FICB has been extensively studied for its efficacy in reducing pain of fracture neck of femur and postoperative pain. A recent Cochrane review that evaluated 8 randomized control trials (RCT) with high level of evidence and included 4 RCTs comparing FICB to systemic analgesics showed reduction in VAS pain score of approximately 3.4 of 10 on movement within 30 min after block placement [8••]. Another meta-analysis including 11 trials and 937 patients has compared the pain scores at various intervals after block versus placebo. As compared with control group, FICB reduced VAS at $1-8 \mathrm{~h}$ with high heterogeneity (standard mean difference $(\mathrm{SMD})=-$ $1.03,95 \%$ confidence interval $(\mathrm{CI})-1.48,0.58, P=0.000$ ). As compared with control, FICB reduced VAS at $12 \mathrm{~h}$ with moderate heterogeneity $(\mathrm{SMD}=-1.06,95 \% \mathrm{CI}-1.36,0.75$, $P=0.000)$ and reduced VAS at $24 \mathrm{~h}$ with high heterogeneity $(\mathrm{SMD}=-1.14,95 \%$ CI $1.66,0.62)[9]$.

\section{Opioid Sparing Effect}

Apart from the benefit of pain control in the FICB group, studies have also shown the opioid sparing effect of the block. Studies comparing FICB to opioids have reported a statistically significant reduction in need for additional analgesia. 
$(\mathrm{SMD}=-1.89,95 \% \mathrm{CI}-3.63,-0.140, P=0.03)[10 \bullet \cdot]$. Also, when compared with systemic analgesia, the FICB group was found to have significantly longer time to first request for additional analgesia. $(\mathrm{SMD}=0.93,95 \%$ CI 0.02 , $1.84, P=0.05)[10 \bullet \cdot$. In studies comparing FICB to NSAIDs, one study which used additional analgesia as outcome reported that the FICB group requested fewer NSAIDs at $21 \%$ compared with $82 \%$ in control group $(P<0.05)$ [11].

\section{Improved Patient Positioning}

The analgesic effect of the block has been analyzed to show reduced pain during positioning for spinal anesthesia. Diakomi et al. compared FICB to intravenous fentanyl (IVFE) and showed that the FICB group had lower numeric pain score rating after intervention $(P<0.001)$, shorter spinal performance time $(P=0.001)$, and better quality of positioning $(P=0.001)[12 \bullet]$.

\section{Effect on Cognition}

Studies have shown a positive effect of FICB on reducing the incidence of delirium, but current level of evidence is deemed to be of low quality and more data is needed to prove this positive outcome $[8 \cdot \bullet, 13]$. Use of regional blocks can play a key role in reduction of opioids and other anxiolytic medications, which can lead to positive effects on cognition of elderly patients.

\section{Nausea}

A recent meta-analysis comparing 6 studies with a total of 224 patients has concluded that patients in FICB group had significantly less occurrence of nausea than in the control group. (Risk ratio $(\mathrm{RR})=0.33,95 \%$ CI $0.17-0.65, P=.001)$. This reduction in nausea could be due to opioid sparing effect of the block [9].

\section{Hospital Length of Stay}

There is some evidence to suggest that patients receiving FICB may have shorter hospital stay after fracture of hip. A study by Callear et al. showed that patients who received a FICB received significantly less postoperative and total analgesia $(P=0.04, P=0.03)$ and had lower rates of delirium $(P=$ 0.03 ), and those patients which were discharged directly home had a shorter inpatient stay $(P=0.03)[14]$.

\section{Femoral Nerve Block: (FNB)}

The femoral nerve is the largest branch of lumbar plexus and supplies sensory fibers to the hip joint apart from motor and sensory supply to the thigh. It is formed by the ventral roots of the L2-L4 spinal nerves. Above the inguinal ligament, the femoral nerve is within the psoas muscle but emerges from the muscle at its middle and lower third junction. It enters the thigh posterior to the inguinal ligament where it is positioned lateral to the femoral artery and lies on the surface of the iliopsoas muscle. All along its course to the thigh, the nerve remains deep to fascia iliaca. The nerve divides into its anterior and posterior branches slightly distal to the inguinal ligament. This nerve can be blocked by landmark, nerve stimulator, or ultrasound guided technique. A single injection or a continuous catheter approach can be employed to block the femoral nerve.

\section{Ultrasound Guided Technique}

A high frequency linear probe is used at the level of the femoral crease with patient lying supine. The femoral artery is identified at this level. If profunda-femoris artery is also seen, the probe is moved proximally to identify the femoral artery before the branching. The femoral nerve lies lateral to the artery and is seen as a hyperechoic triangular or oval structure covered by a hyperechoic fascia iliaca (Fig. 1). The needle is typically inserted in an in-plane technique from lateral to medial direction, though an out of plane injection with needle insertion perpendicular to probe can also be made (Fig. 1b). Using the in-plane technique, once the needle tip is lateral or above or below the nerve, an injection is made to see the spread of LA. Continuous nerve catheter can also be threaded under ultrasound guidance for prolonged postoperative analgesia. A nerve stimulator can also be simultaneously used along with ultrasound guidance to confirm the location of femoral nerve by eliciting quadriceps/patellar twitch. This dual guidance has not shown any improved success rate over the stand-alone ultrasound guided technique, but may be helpful in preventing neurological injury.

\section{3 in 1 Block}

Winnie et al. first described the 3 in 1 block in 1973. They postulated that the entire lumbar plexus can be blocked by a single perivascular injection slightly distal to the inguinal ligament (location of femoral nerve in femoral triangle). They also suggested that this single injection would result in anesthesia of the femoral, lateral femoral cutaneous (LFCN), and obturator nerves $(\mathrm{ON})$ due to cephalad distribution of local anesthetic along the fascial layer and thereby blocking the nerves of the lumbar plexus. This hypothesis was never confirmed and subsequent; MRI studies have shown distribution of local anesthetic that follows a lateral and medial spread after injection and not a cephalad spread.

One study proposed that the term 3 in 1 block should be abandoned since it rarely blocks the obturator nerve [15]. It has been previously suggested that the block be better referred 
to as the 2 in 1 block [16]. The 3 in 1 block as classically described is performed at the same site as femoral nerve block with additional application of pressure $2-4 \mathrm{~cm}$ distal to the needle held for $30 \mathrm{~s}$ and a larger volume of LA. Landmarkbased technique is now outdated, and currently this block is performed by nerve stimulator or ultrasound guided technique. The anteromedial hip joint is supplied by articular braches of the ON, and the LFCN provides sensory innervation to lateral thigh. The LFCN and ON can be blocked independently with ultrasound guidance along with FNB instead of a 3 in 1 block for hip analgesia. Since some literature still describe femoral block as 3 in 1 block, for the purpose of this article, results of studies using either femoral or 3-1 blocks were combined since these two blocks are variants of each other with minor differences.

\section{Analgesic Efficacy}

The Cochrane review that evaluated 8 RCTs with high level of evidence included 4 RCTs, which used FNB for hip fracture. The review suggested a reduction in VAS pain score within 30 min after block placement of approximately 3.4 on a scale of 0 to 10 on movement $(\mathrm{SMD}=-1.41,95 \%$ CI 2.14 to $0.67, P=0.02)[8 \cdot \bullet]$. Another systemic review by Abou-Setta found that FNB provided superior analgesia $(P<0.001)$ as compared with no block or standard care [17]. Another meta-analysis by Skjold et al. showed significantly decreased VAS pain scores by 2.13 points in intervention group as compared with control group $(\mathrm{CI},-3.53,-0.72)$ [18].

\section{Opioid Sparing Effect}

Morrison et al. performed a RCT comparing effect of single dose femoral block in emergency department (ED) followed by a continuous FICB by anesthesiologist within $24 \mathrm{~h}$ vs conventional analgesics [19॰]. The results in the ED were in favor of intervention with these patients receiving 33\% fewer parenteral morphine sulfate equivalents than control, with doses of $0.8 \mathrm{mg} / \mathrm{h}(95 \%$ CI $0.64,1.05)$ versus $1.2 \mathrm{mg} / \mathrm{h}(95 \%$ CI $0.94,1.40)$, respectively.

\section{LAST (Local Anesthetic Systemic Toxicity)}

Ultrasound guided nerve blocks enable visualization of deposition of local anesthetic in the correct plane, prevent intravascular injection and thus LAST. Elderly patients are sensitive to LA doses, and ultrasound guidance enables reduction of the total dose of LA and therefore prevention of LAST.

\section{Time to First Mobilization}

Based on recent Cochrane review, which included 2 trials with 155 participants (femoral nerve block and obturator nerve block with or without lateral femoral cutaneous nerve block), peripheral nerve blocks reduced time to first mobilization with a mean difference of $-11.25 \mathrm{~h}(95 \% \mathrm{CI},-14.34 \mathrm{to}-8.15 \mathrm{~h})$ $[8 \bullet \bullet]$. The quality of evidence for this result was rated as moderate. This early mobilization may have a beneficial role in the prevention of deep vein thrombosis.

\section{Pneumonia}

Based on the premise of improved analgesia and reduced time to first mobilization in patients with FNB, a reduction in incidence of pneumonia has been shown in two trials, one by Fletcher et al. and other by Haddad et al., which have been included in the Cochrane review on nerve blocks for hip fracture $[8 \cdot \bullet]$.

\section{Delirium}

There have been some studies suggesting reduced incidence of delirium in patients receiving FNB for hip fracture [16, 20], but the Cochrane review by Guay et al. was unable to identify adequate high-quality evidence to demonstrate reduction in incidence of delirium in patients receiving a femoral nerve block $[8 \cdot \bullet]$.

\section{Mortality}

There has been some suggestion that use of nerve block may reduce the incidence of mortality after hip fracture, but the evidence is lacking. The Cochrane review by Guay et al. included 5 trials out of 7 ( $4=$ femoral nerve block or 3 -in- 1 block, $1=$ femoral nerve block plus infiltration above iliac crest). This review did not find any difference in short-term mortality (6 months): RR, 0.72 (95\% CI, 0.34-1.52); I ${ }^{2}$ statistics $=0 \%[8 \cdot \bullet]$.

\section{Lumbar Plexus Block: (Psoas Compartment Block)}

The hip receives it sensory supply from the lumbar plexus, which lies within the psoas muscle. Hence, block of the lumbar plexus is a regional technique, which has been employed for not only analgesia but also anesthesia for surgery for hip fracture [21]. Lumbar plexus block/psoas compartment block is a technically difficult deep block that requires greater skill and hence can be time consuming in the hands of the novice. The lumbar paravertebral region is highly vascular and noncompressible. Hence, patients on anti-coagulation are at high risk for bleeding complications. There is also high risk of unintentional neuraxial block or inadvertent intravascular injection with local anesthetic systemic toxicity [22]. Traditionally, it is performed with anatomical landmarks technique, while its performance with dual ultrasound guidance and peripheral nerve stimulation can decrease the 
complications and increase the success rate. Psoas compartment block is gone out of favor in clinical use currently due to growing use of easy and safe alternatives blocks like FICB and FNB.

\section{Novel Interfascial Plane Blocks}

\section{Quadratus Lumborum Block (QLB) and Erector Spinae Plane Block (ESP)}

Lumbar QLB and ESP are abdominal interfascial plane blocks where the LA is deposited in a fascial plane surrounding the quadratus lumborum muscle (QLM) or erector spinae muscle (ESM) to block the nerves. QLB is classified into lateral, posterior, and anterior (transmuscular) types based on location of LA placement in relation to the QLM and other abdominal muscles [23•]. In ESPB, the LA is deposited deep to the ESM on the top of transverse process (TP). Few case reports and studies of posterior QLB and anterior QLB (RCT) have shown reduced pain scores, reduced opioid consumption, and improved patient satisfaction for primary total hip arthroplasty [24]. Transmuscular QLB reduced hospital length of stay for patients undergoing total hip arthroplasty [25]. Another study comparing lumbar ESPB, QL, and control groups showed improved analgesic quality with ESPB and QLB groups in patients undergoing hip and proximal femoral surgery [26]. However, some nerve braches responsible for hip joint innervation may not be blocked by QLB or ESPB, which should be taken into consideration while using these blocks for hip fracture.

\section{PEricapsular Nerve Group Block: (PENG Block)}

Articular branches of femoral, obturator, and accessory obturator nerves (AON) supply the hip joint. The PENG block is an interfascial plane block aiming to block these articular branches to enable hip analgesia.

\section{Ultrasound Guided Technique}

A low frequency curvilinear transducer is placed in transverse plane over the anterior inferior iliac spine (AIIS) and moved over inferiorly to visualize the pubic ramus (Fig. 4). The femoral artery and ilio-pubic eminence (IPE) are then visualized (Fig. 4b). Using in-plane needling technique from lateral to medial direction, LA is deposited between the psoas tendon anteriorly and pubic ramus posteriorly. Usually, 20-25 $\mathrm{ml}$ of LA is used for this block.

A small case series published along with the description of this novel PENG block showed good analgesic benefit for hip fractures. The median reduction of pain scores in this study was 7 points, showing a larger decrease in pain scores compared with other regional techniques in hip fractures [27••].
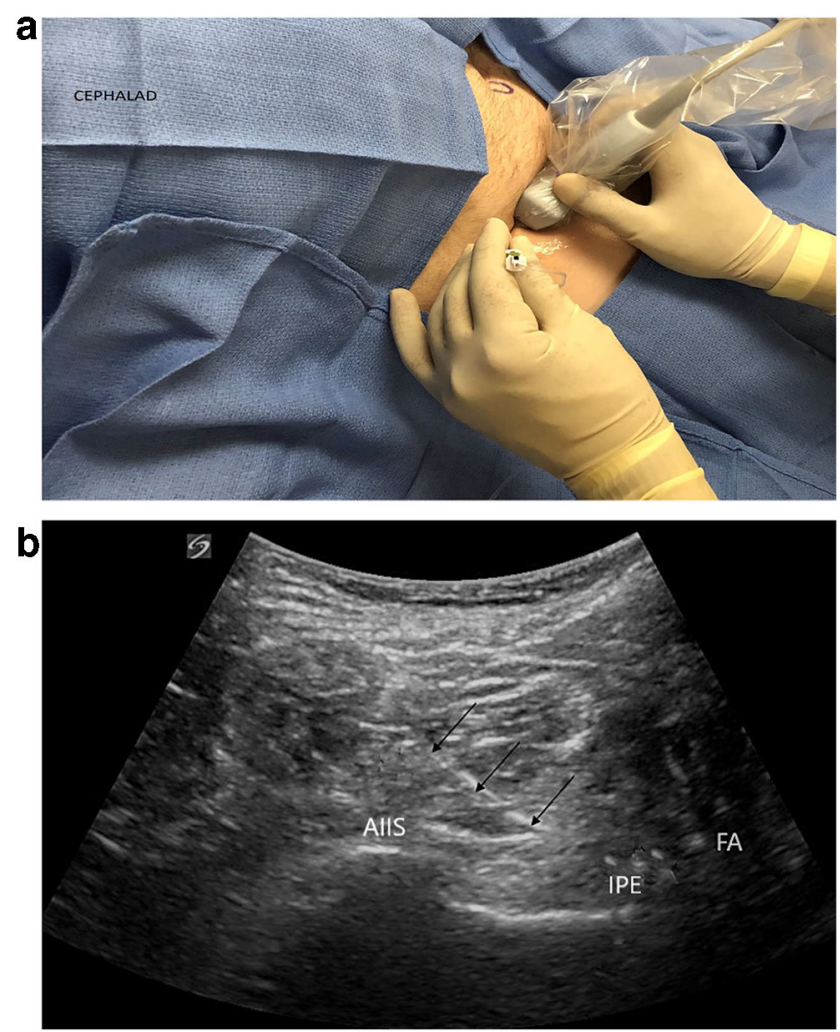

Fig. 4 a PEricapsular Nerve Group Block (PENG Block): A curvilinear probe placed transversely over the anterior inferior iliac spine (AIIS), and it is then aligned with pubic ramus. Needle is inserted in-plane from lateral to medial. b Ultrasound image of PENG block showing lateral to medial insertion of needle (black arrows) showing the anterior inferior iliac spine (AIIS), ilio-pubic eminence (IPE), and femoral artery (FA)

The benefits of the PENG block are patient positioning for procedure, no significant motor weakness (potential motor sparing effect), and analgesic efficacy [28]. The disadvantage is that it cannot be used as a sole anesthetic block for the hip surgery, though it can used in combination with other nerve blocks like FIB/FICB for more extensive analgesia for hip surgery. The PENG block and lateral femoral cutaneous nerve block (LFCN) combinations have also been used for hip surgery analgesia.

\section{Conclusions}

Ultrasound guided FICB can be performed either as an infrainguinal FICB and supra-inguinal FICB. The FICB has shown good analgesic efficacy for hip fracture patients with SIFICB showing superior results than other approaches due to better spread of LA to all the nerves. FICB is considered as an easy and safe alternative and an anterior approach to the lumbar plexus with positive outcomes like opioid sparing effect, improved patient positioning and less nausea. FNB is also extensively used for hip fracture analgesia in most centers with good beneficial effects like reduced pain scores, reduced time 
to first mobilization, lower incidence of delirium, and pneumonia. Lumbar plexus block is a deep block, and is difficult to perform and due to associated complications, it has gone out of favor in clinical practice now and replaced by FICB. The PENG block is now more extensively used and studied for hip fracture pain with positive evidence from few case reports. Other blocks like QLB and ESP can play a role in hip surgery analgesia in addition to the hip fracture analgesia, and studies have currently shown the benefits of these blocks for total hip arthroplasty. Regional analgesia along with neuraxial anesthesia for hip fracture surgery has a multitude of benefits with positive outcomes and will play a major role in the pain and perioperative management of these patients in the future. Peripheral nerve blocks like FICB, FNB, and PENG blocks should be strongly considered for elderly patients for hip fracture analgesia.

Open Access This article is licensed under a Creative Commons Attribution 4.0 International License, which permits use, sharing, adaptation, distribution and reproduction in any medium or format, as long as you give appropriate credit to the original author(s) and the source, provide a link to the Creative Commons licence, and indicate if changes were made. The images or other third party material in this article are included in the article's Creative Commons licence, unless indicated otherwise in a credit line to the material. If material is not included in the article's Creative Commons licence and your intended use is not permitted by statutory regulation or exceeds the permitted use, you will need to obtain permission directly from the copyright holder. To view a copy of this licence, visit http://creativecommons.org/licenses/by/4.0/.

\section{References}

Papers of particular interest, published recently, have been

highlighted as:

- Of importance

•- Of major importance

1. Lewiecki EM, Wright NC, Curtis JR, Siris E, Gagel RF, Saag KG, et al. Hip fracture trends in the United States, 2002 to 2015. Osteoporos Int. 2018;29:717-22.

2. Singer A, Exuzides A, Spangler L, et al. Burden of illness for osteoporotic fractures compared with other serious diseases among postmenopausal women in the United States. Mayo Clin Proc. 2015;90:53-62.

3. Mears SC, Kates SL. A guide to improving the care of patients with fragility fractures, edition 2. Geriatr Orthop Surg Rehabil. 2015;6(2):58-120.

4. Birnbaum K, Prescher A, Hessler S, Heller KD. The sensory innervation of the hip joint - an anatomical study. Surg Radiol Anat. 1997;19(6):371-5.

5. Hebbard P, Ivanusic J, Sha S. Ultrasound-guided supra-inguinal fascia iliaca block: a cadaveric evaluation of a novel approach. Anaesthesia. 2011;66:300-5.

6. Desmet M, Vermeylen K, Van Herreweghe I, et al. A longitudinal supra-inguinal fascia iliaca compartment block reduces morphine consumption after total hip arthroplasty. Reg Anesth Pain Med. 2017;42:327-33.

7. Vermeylen K, Matthias D, Leunen I, et al. Supra-inguinal injection for fascia iliaca compartment block results in more consistent spread towards the lumbar plexus than an infra-inguinal injection: a volunteer study. Reg Anesth Pain Med. 2019;44:483-91 A RCT showing supra-inguinal injection for fascia iliaca block having reliable spread of local anesthetic to the three target nerves of the lumbar plexus on MRI as compared to infra-inguinal approach.

8.• Guay J, Parker MJ, Griffiths R, Kopp SL. Peripheral nerve blocks for hip fractures: a Cochrane Review. Anesth Analg. 2018;126(5): 1695-704 A Cochrane review which focuses on peripheral nerve blocks for pre or post-operative analgesia for hip fracture surgery.

9. Hong HK, Ma Y. The efficacy of fascia iliaca compartment block for pain control after hip fracture: a meta-analysis. Medicine (Baltimore). 2019;98(28):e16157.

10.• Steenberg J, Moller AM. Systemic review of the effects of fascia iliaca compartment block on hip fracture patients before operation. Br J Anaesth. 2018;120(6):1368-80 Systematic review of FICB, which concludes it as a relatively safe and effective supplement in perioperative pain management of hip fracture patients.

11. Fujihara Y, Fukunishi S, Niship S, Miura J, Koyanagi S, Yoshiya S. Fascia iliaca compartment block: its efficacy in pain control for patients with proximal femoral fracture. J Orthop Sci. 2013;18: 793-7.

12. Diakomi M, Papaiommou M, Mela A, Kouskouni E, Makris A. Preoperative fascia iliaca compartment block for positioning patients with hip fractures for central nervous system blockade: a randomized trial. Reg Anesth Pain Med. 2014;39:394-8 An RCT comparing FICB with IV opioids, which shows FICB enabling satisfactory postoperative analgesia and improved patient acceptance compared to systemic opioids.

13. Mouzopoulos G, Vasiliadis G, Lasanianos N, Nikolaras G, Morakis E, Kaminaris M. Fascia iliaca block prophylaxis for hip fracture patients at risk for delirium: a randomized placebo controlled trial. J Orthop Traumatol. 2009;10:127-33.

14. Callear J, Shah K. Analgesia in hip fractures. Do fascia-iliac blocks make any difference? BMJ Qual Improv Rep. 2016;5(1): u210130.w4147. https://doi.org/10.1136/bmjquality.u210130. w4147.

15. Moore CL. Time to abandon the term "3 in 1 block". Ann Emerg Med. 2015;66(2):215.

16. Lang SA, Yip RW, Chang PC, et al. The femoral 3 in 1 block revisited. J Clin Anesth. 1995;39:292-6.

17. Abou-Setta AM, Beaupre LA, Rashiq S, Dryden DM, Hamm MP, Sadowski CA, et al. Comparative effectiveness of pain management interventions for hip fracture: a systemic review. Ann Intern Med. 2011;155:234-45.

18. Skjold C, Moller AM, Wildgaard K. Pre-operative femoral nerve block for hip fracture - a systemic review with meta-analysis. Acta Anaesthesiol Scand. 2020;64:23-33.

19. Morrison RS, Dickman E, Hwang U, et al. Regional nerve blocks improve pain and functional outcomes in hip fracture: a randomized control trial. J Am Geriatr Soc. 2016;64:2433-9 RCT comparing outcomes of regional nerve block with that of standard analgesics after hip fracture.

20. Del Rosario E, Esteve N, Sernandez MJ, Batet C, Aguilar JL. Does femoral nerve analgesia impact the development of postoperative delirium in the elderly? A retrospective investigation. Acute Pain. 2008;10:59-64.

21. Ahamed ZA, Sreejot MS. Lumbar plexus block as an effective alternative to subarachnoid block for intertrochanteric hip fracture surgeries in the elderly. Anesth Essays Res. 2019;13(2):264-8. 
22. Zhang XH, Li YJ, He WQ, Yang CY, Gu JT, Lu KZ, et al. Combined ultrasound and nerve stimulator guided deep nerve block may decrease the rate of local anesthetics systemic toxicity: a randomized clinical trial. BMC Anesthesiol. 2019;19:103.

23. Gupta A, Sondekoppam R, Kalagara H. Quadratus Lumborum Block: A Technical Review. Curr Anesthesiol Rep. 2019;9:25762 A technical description of various types of QLB and its current clinical uses and evidence.

24. Kukreja P, MacBeth L, Sturdivant A, et al. Anterior quadratus lumborum block analgesia for total hip arthroplasty: a randomized, controlled study. Reg Anesth Pain Med. 2019;44:1075-9.

25. Stuart Green M, Ryan Hoffman C, Iqbal U, Olabisi Ives O, Hurd B. Transmuscular quadratus lumborum block reduces length of stay in patients receiving total hip arthroplasty. Anesth Pain Med. 2018;8(6):e80233.

26. Tulgar S, Kose HC, Selvi O, et al. Comparison of ultrasoundguided lumbar erector spinae plane block and transmuscular quadratus lumborum block for postoperative analgesia in hip and proximal femur surgery: a prospective randomized feasibility study. Anesth Essays Res. 2018;12(4):825-31.

27.• Girón-Arango L, Peng PW, Chin KJ, et al. Pericapsular Nerve Group (PENG) block for hip fracture. Reg Anesth Pain Med. 2018;43:859-63 A report describing technique and application of novel ultrasound guided approach to block the articular branches to the hip by PENG block.

28. Ueshima H, Otake H, et al. Clinical experiences of pericapsular nerve group (PENG) block for hip surgery. J Clin Anesth. 2018;51:60-1.

Publisher's Note Springer Nature remains neutral with regard to jurisdictional claims in published maps and institutional affiliations. 